\title{
Ultrasonography and Color Doppler in the Differential Diagnosis of Periapical Cyst and Granuloma
}

\author{
${ }^{1}$ Brijesh Tiwari, ${ }^{2}$ Manu Krishnan, ${ }^{3}$ Manju Popli, ${ }^{4}$ Satish R lyer, ${ }^{5}$ Vimal Arora
}

\begin{abstract}
Background and objectives: Though ultrasonography and color Doppler are well established diagnostic techniques in medical sciences, it is not so popular in dentistry. Aim of the study was to evaluate the diagnostic value of ultrasonography and color Doppler in periapical cysts and granulomas in the anterior region of the jaws.
\end{abstract}

Materials and methods: Thirty patients aged between 30 and 50 years with periapical lesions in anterior teeth were selected and consented for ultrasonography and color Doppler examinations. Shape, boundary, echo intensity, echo pattern, acoustic enhancement and vascularity of the periapical lesions and adjacent tissues were studied. After endodontic filling, lesions were enucleated and histopathology was done. Ultrasonographic/color Doppler findings and histopathology were statistically evaluated with Chi-square tests at $p<0.001$. Sensitivity, specificity, positive and negative predictive values and accuracy of the method were then determined.

Results: Noninfected cysts showed as anechoic areas while infected cysts appeared as hypoechoic. Vascular hypoechoic areas proved to be periapical granulomas. Periapical infected and noninfected cysts maintained 100\% correlation with histopathology, while periapical granuloma had only $70 \%$ relation due to thick overlying cortical bones. It implied an accuracy of $90 \%$ for this method with overall positive and negative predictive values of $86.96 \%$ and $100 \%$ respectively.

Conclusion: The study demonstrated efficacy of USG in combination with color Doppler as a safe, noninvasive option to diagnose solid/cystic periapical lesions. However, it also highlighted the need for further investigations in resolving diagnostic issues of periapical lesions covered with thick cortical bones.

Keywords: Ultrasonography, Color Doppler, Periapical cyst, Periapical granuloma.

How to cite this article: Tiwari B, Krishnan M, Popli M, lyer $\mathrm{SR}$, Arora V. Ultrasonography and Color Doppler in the

\footnotetext{
${ }^{1}$ Senior Research Fellow, ${ }^{2} \mathrm{Head}$ and Classified Specialist ${ }^{3}$ Scientist ' $F$ ' and Joint Director, ${ }^{4}$ Deputy Director General Dental Services and Classified Specialist, ${ }^{5}$ Director General Dental Services and Classified Specialist

1,2Department of Dental Research and Implantology, INMAS DRDO, Delhi, India

${ }^{3}$ Department of Radiological Imaging, INMAS, DRDO Delhi, India

${ }^{4,5}$ Directorate General Dental Services, IHQ of MoD (Army) New Delhi, India
}

Corresponding Author: Brijesh Tiwari, Senior Research Fellow, Department of Dental Research and Implantology INMAS, DRDO, Delhi, India, Phone: 01123939588, e-mail: inmasdentalresearch@gmail.com
Differential Diagnosis of Periapical Cyst and Granuloma. J Contemp Dent 2014;4(1):17-21.

Source of support: Nil

Conflict of interest: None

\section{INTRODUCTION}

Periapical granuloma and cyst are consequent to the inflammatory response of the tooth pulp to various irritants. Granuloma is a mass of vascular granulation tissue formed at the apex of a nonvital tooth and is indeed an attempt to prevent the spread of harmful toxic products to surrounding tissues and thereby to systemic circulation. Granuloma can progress into a periapical (radicular) cyst; the most common odontogenic cyst or into a periapical abscess. ${ }^{1}$ A cyst may warrant surgical endodontics more frequently as compared to granuloma. ${ }^{2}$ Therefore, correct diagnosis of periapical cyst and granuloma can be decisive in opting for invasive surgical or nonsurgical endodontics and is therefore clinically important. ${ }^{3}$

Diagnostic value of ultrasound imaging in all the disciplines of medicine and surgery has been well established. ${ }^{4}$ Ultrasonography (USG) uses high frequency sound waves in the range of 2 to $18 \mathrm{MHz}$ generated from a transducer/probe. These nonaudible waves travel at different speeds through different media and are absorbed, reflected or scattered based on their acoustic impedance values. The echoes are then sent back to the probe where they are detected, amplified and analyzed to form the image. Different body tissues produce different echo intensities to form the USG images. Calcified structures like bone which are highly reflective to ultrasound waves appear white (hyperechoic), while fluids that transmit ultrasound waves are seen as black (anechoic). Soft tissues with densities in between these two extremes present as different shades of gray (hypoechoic). ${ }^{5}$ When observing vascular tissues, sonography is usually augmented with color Doppler. ${ }^{6}$

An early use of ultrasonography in dentistry was for detecting the dentin-enamel and dentin-pulp interface of teeth. ${ }^{7}$ It was then used for periodontal disease assessment, ${ }^{8}$ temporomandibular joint imaging, ${ }^{9}$ mandibular fracture ${ }^{10}$ and intraosseous lesions of the jaws. ${ }^{2,11}$ Its utility in 
diagnosing periapical lesions either alone ${ }^{2}$ or in combination with color Doppler ${ }^{11-15}$ have generated renewed interest among clinicians; being a safe and noninvasive technique. However, in spite of its promising reports in delineating periapical lesions, it has not become a popular method in contemporary clinical practice. Differences in the nature and thickness of jaw bones at different facial sites are unfavorable for applying USG with uniform results. The increased thickness of posterior jaw bones; for both the maxilla and mandible, act as a deterrent in imaging respective root apices with ultrasound waves. ${ }^{16}$ In this context, it is imperative to evaluate whether ultrasound imaging can be used to diagnose periapical granulomas and cysts with reliable sensitivity and specificity in the anterior jaw areas.

\section{MATERIALS AND METHODS}

Thirty patients aged between 30 and 50 years diagnosed clinically and radiologically with periapical lesions, in the maxillary or mandibular anterior teeth, were selected for the study. To ensure that lesions were inflammatory and exclusively pulpal in origin, only those patients with caries exposed or nonvital tooth with history of trauma were included, after an informed consent and clearance from institutional ethics committee.

Ultrasonography was carried out with a linear array, regular-size probe at a frequency of $10 \mathrm{MHz}$; Vivid 7 ultrasound system, GE Medical, WI, USA. Probe was placed extraorally over the apical area of the affected tooth for ultrasonography and color Doppler examination. The echo intensity anechoic/hypoechoic, internal echo pattern, posterior echo enhancement, contour, boundary and vascularity of the lesions were used to describe the lesions. ${ }^{4}$ After root canal filling, endodontic surgery was performed under local anesthesia in aseptic conditions. Periapical lesions were then completely excised; tissue was cleaned with normal saline and was kept in $10 \%$ formalin solution. Samples were processed and stained with hematoxylin and eosin stain and were examined under light microscope (Olympus light microscope, Olympus Corporation, Tokyo, Japan) at $\times 100$ magnification.

Association between USG and histopathology was analyzed with Chi-square tests. Sensitivity, specificity, positive and negative predictive values and accuracy of USG and color Doppler in periapical lesions were also determined.

\section{RESULTS}

Out of the 30 cases, USG and color Doppler showed presence of periapical lesions in 27 cases (Table 1). Five lesions presented with anechoic areas with posterior echo enhancement and increased echo intensity at bony contours. They had well-defined, round, smooth contours with no evidence of internal vascularity in color Doppler. These cases were ultrasonographically diagnosed as non- infected periapical cysts (Fig. 1). Its histopathology also correlated to USG and color Doppler diagnosis (Fig. 2). The samples showed connective tissue walls with thin layer of stratified squamous epithelium lining the cavity. In cases of intense inflammation, epithelium was either not seen or was discontinuous. Contents of the cyst looked pale eosinophilic, suggestive of its low protein concentration. Fibroblasts, plasma cells and lymphocytic infiltration were other cellular types.

Fifteen lesions showed hypoechoic areas with heterogeneous internal echo pattern. They had increased echo intensity at marginal bony contours which were mostly irregular. Lesions showed round or variable shape, with no vascularity in the color Doppler. Changes in the echo intensity of the surrounding soft tissues were also noteworthy. These cases were diagnosed as infected periapical cysts by USG (Fig. 3) and were later confirmed by histopathology also. Samples had high degrees of inflammatory infiltrate, even in the lumen of the cysts with absence of epithelial lining (Fig. 4).

Seven cases were seen with hypoechoic areas and poorly defined borders in USG and rich internal vascularity in color Doppler (Fig. 5). They were diagnosed as periapical granulomas. Histopathology reaffirmed USG and color

Table 1: Association between USG/color Doppler findings and histopathology of periapical lesions

\begin{tabular}{|c|c|c|c|c|}
\hline \multirow[t]{2}{*}{ USG/color Doppler diagnosis } & \multicolumn{3}{|c|}{ Histopathologic diagnosis } & \multirow[t]{2}{*}{ Total } \\
\hline & Noninfected cyst & Infected cyst & Granuloma & \\
\hline \multirow[t]{2}{*}{ Normal tissue } & & & 3 & 3 \\
\hline & & & $30 \%$ & $10 \%$ \\
\hline \multirow[t]{2}{*}{ Noninfected cyst } & 5 & & & 5 \\
\hline & $100 \%$ & & & $16.70 \%$ \\
\hline \multirow[t]{2}{*}{ Infected cyst } & & 15 & & 15 \\
\hline & & $100 \%$ & & $50 \%$ \\
\hline \multirow[t]{2}{*}{ Granuloma } & & & 7 & 7 \\
\hline & & & $70 \%$ & $23.30 \%$ \\
\hline Total & 5 & 15 & 10 & 30 \\
\hline
\end{tabular}


Doppler observations (Fig. 6). Tissue samples showed numerous fibroblasts, connective tissue fibrils and vascular channels. Other cell elements included macrophages, lymphocytes, plasma cells, mast cells and multinucleated giant cells.

Rest three cases showed no periapical lesion on USG and color Doppler but proved to be periapical granuloma in the histopathology. Relation between USG/color Doppler of periapical lesions with histopathology is shown in Table 1. Infected and noninfected cysts demonstrated 100\% correlation, while periapical granuloma had $70 \%$ association at $\mathrm{p}<0.001$. This related to $90 \%$ accuracy with overall positive and negative predictive values of $86.96 \%$ and $100 \%$ respectively, for USG and color Doppler in diagnosing dental periapical lesions (Table 2 and Graph 1).

\section{DISCUSSION}

In the present study, we used a B-mode USG for diagnosing the periapical lesions. In this mode, ultrasound waves are

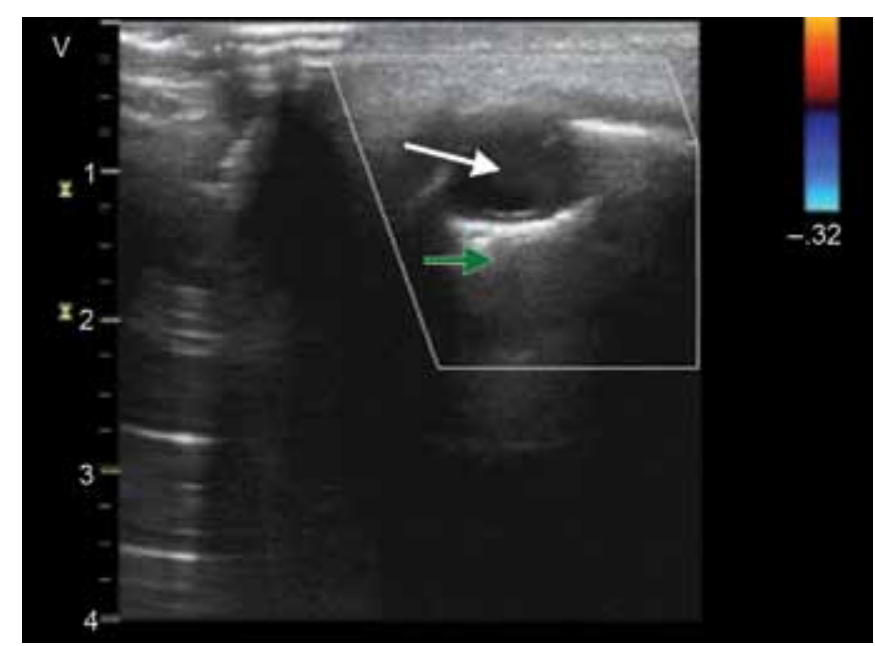

Fig. 1: USG and color Doppler image of 'noninfected' periapical cyst; white arrow shows decreased echo intensity, while green showing posterior echo enhancement

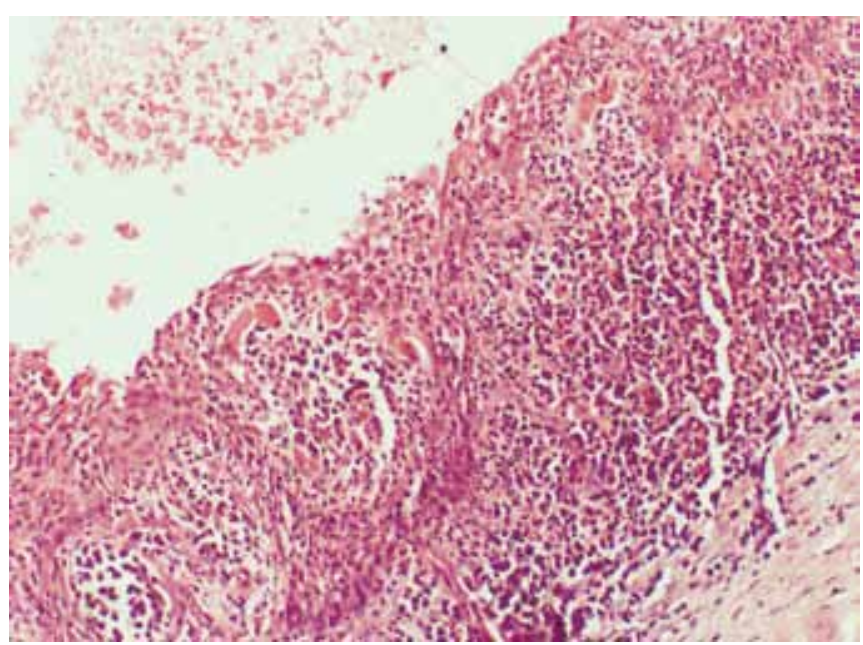

Fig. 2: Histopathology of periapical cyst swept through the patient's body producing a 2D image in the form of tiny dots of different shades of gray. Both static and real time images are possible in this mode with provision for applying color Doppler. The probes used in USG are also of different types: low frequency curved probe, high frequency linear array probe and phased array microconvex probe. Linear array probes operate at high frequency and produce a rectangular image of high resolution. They are effective for scanning relatively superficial musculoskeletal structures ${ }^{4}$ and therefore we used it for dental periapical lesions. We observed that the USG imaging were $100 \%$ effective for cysts and $90 \%$ for granulomas. Its high diagnostic accuracy is worth comparing against the deleterious effects of radiation involved in conventional dental imaging. Globally, dental X-rays constitute $15 \%$ (480 million) of the total diagnostic X-ray examinations done every year. ${ }^{17}$ Panoramic examination has an effective dose of 3-11 microsieverts $(\mu \mathrm{Sv})$ while cephalograms have 5 to $7 \mu \mathrm{Sv}$, occlusal $5 \mu \mathrm{Sv}$, bite wing 1-4 $\mu \mathrm{Sv}$, full mouth intraoral periapical

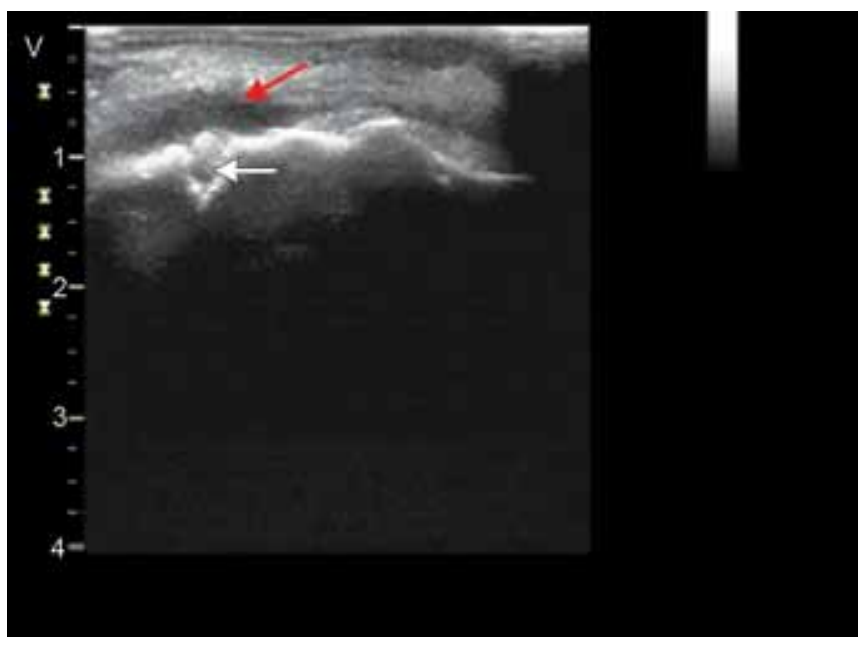

Fig. 3: USG and color Doppler image of 'infected' periapical cyst; white arrow shows heterogeneous internal echo pattern and irregular contour while red arrow showing changes in echo intensity of the surrounding tissues corroborating spread of infection

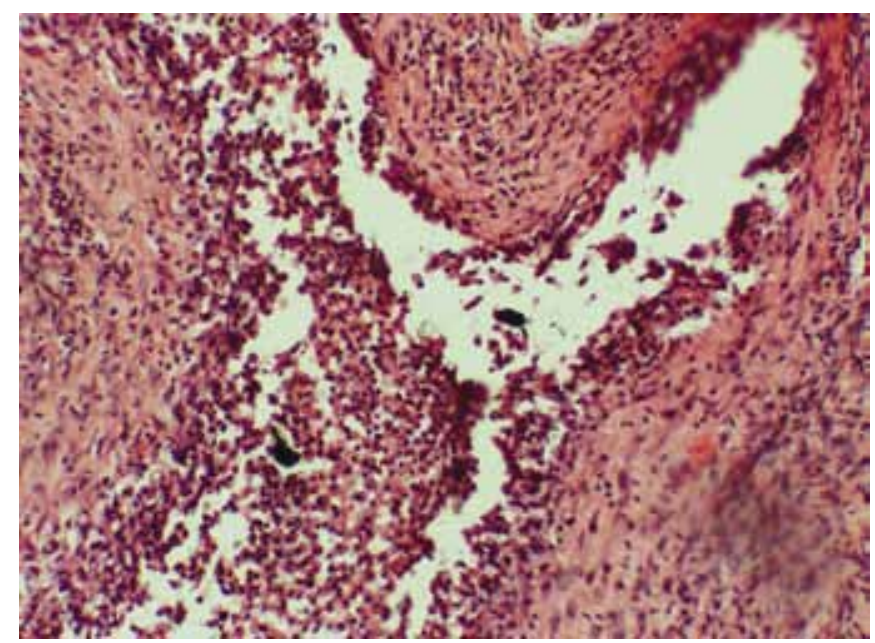

Fig. 4: Histopathology of infected periapical cyst 
30 to $170 \mu \mathrm{Sv}$, TMJ series 20 to $30 \mu \mathrm{Sv}$ and 40 to $135 \mu \mathrm{Sv}$ for cone beam computed tomography $(\mathrm{CBCT}) .{ }^{18}$ In contrast, USG and color Doppler, being acoustic in nature, are radiation free and without any adverse effects.

In a major study of 23 intraosseous lesions in the jaw using ultrasonography, Lauria et $\mathrm{al}^{2}$ reported anechoic pattern in 17 noninfected cases. Two infected cysts could not be rightly identified in their work. Other four went wrong due to thick vestibular bone plate. In our investigation, all

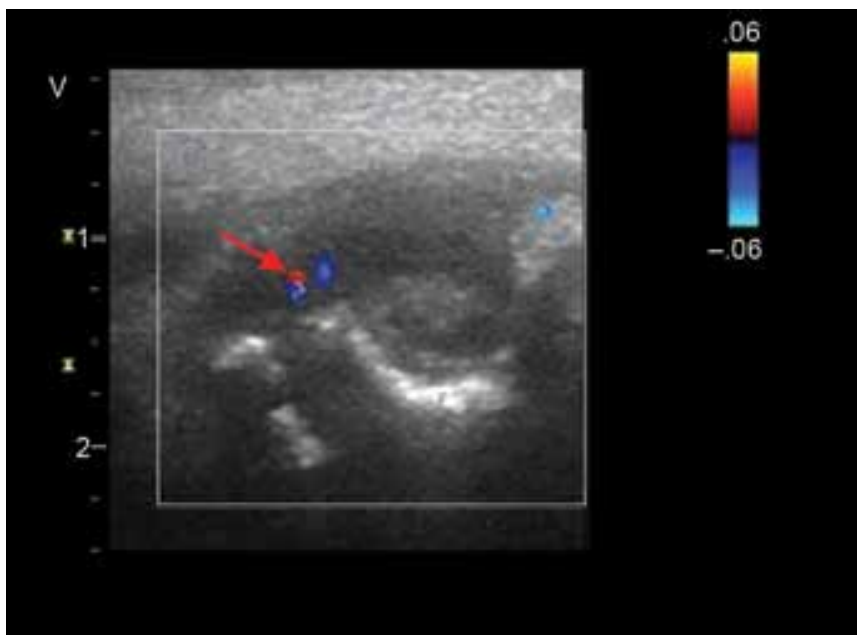

Fig. 5: USG and color Doppler image of periapical granuloma shown in red arrow as poorly defined hypoechoic lesion with rich internal vascularity

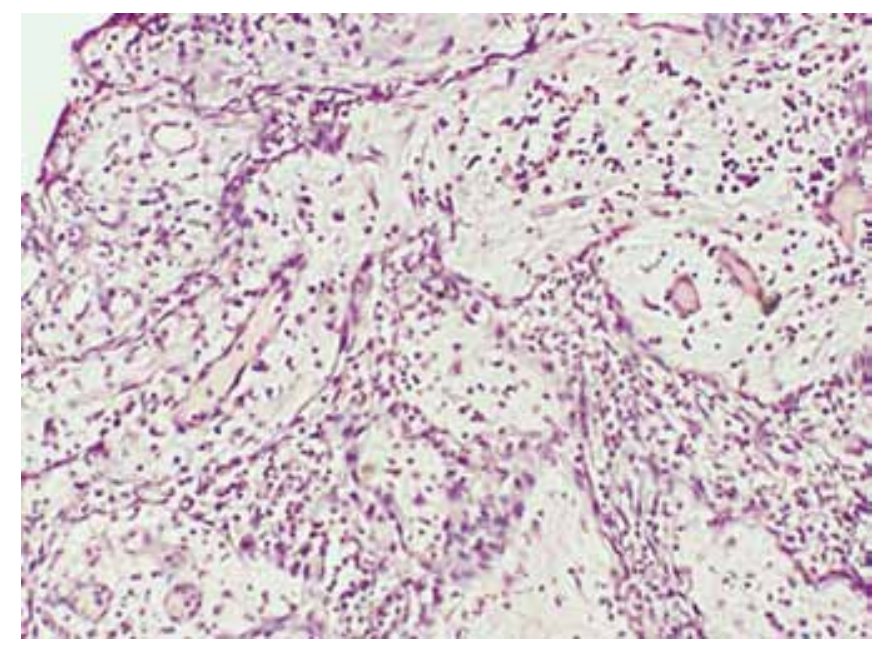

Fig. 6: Histopathology of periapical granuloma the cystic cases, both noninfected and infected, showed definitive anechoic and hypoechoic patterns respectively. We focused both the lesion and surrounding tissues and used color Doppler to access the rich vascularity in periapical granulomas. However, we could not identify three periapical granulomas correctly, due to the thick bone overlying the lesion in those cases, highlighting its role in obscuring jaw USG imaging.

Application of color Doppler in USG for diagnosing periapical cysts and granuloma has been previously reported by Cotti et $\mathrm{al}^{12}$ and Gundappa et al. ${ }^{14}$ Former had 11 cases, while latter reported experience of 15 patients. Both the reports showed $100 \%$ correlation between USG and histopathology. Similar results have been reported by Goel et $\mathrm{al}^{15}$ also. When studying 30 cases, we, however, could only get $90 \%$ association between granuloma and its histopathology. Missing of diagnosis in periapical granuloma was because of the thick buccal cortical bone overlying the lesions. This became evident on the surgical exploration during endodontic surgery. It follows that further investigations and USG parameters are required for rightly describing periapical granulomas and cysts with thick cortical bones.

The thickness of cortical plate varies in maxilla and mandible and even at different locations on the same

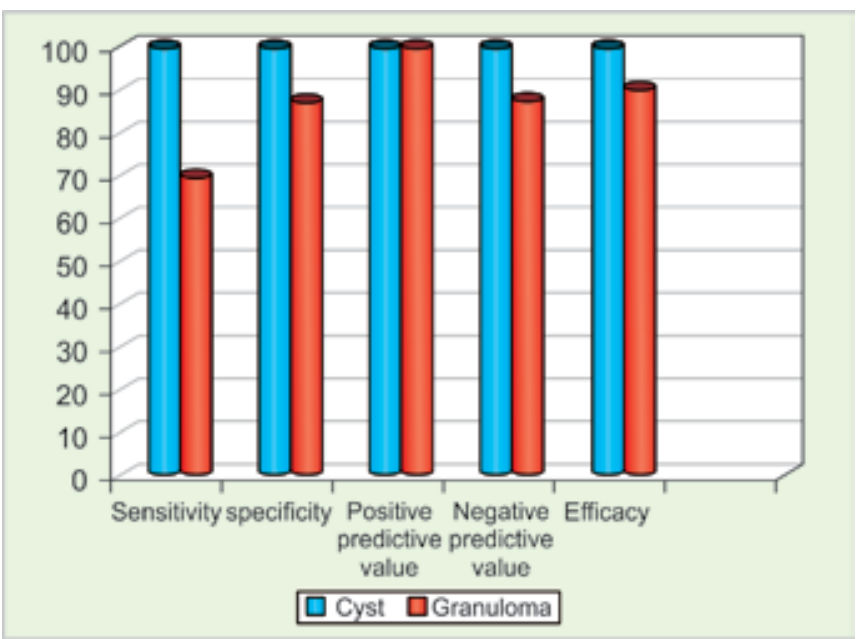

Graph 1: Diagnostic value of USG and color Doppler for periapical cyst and granuloma

Table 2: Diagnostic accuracy of periapical cysts and granuloma with USG/color Doppler

\begin{tabular}{lllll}
\hline Periapical lesion & True positive $(n)$ & True negative $(n)$ & False positive $(n)$ & False negative $(n)$ \\
\hline Periapical cysts & 20 & 10 & 0 & 0 \\
Periapical granuloma & 7 & 20 & 0 & 3 \\
\hline & & Overall diagnostic value & \\
\hline & Sensitivity & $100 \%$ \\
& Specificity & $70 \%$ \\
& Positive predictive value & $86.96 \%$ \\
& Negative predictive value & $100 \%$ & $90 \%$ \\
& Accuracy & & \\
\end{tabular}


bones. Mandibular cortical thickness decreases from $3.7 \mathrm{~mm}$ in the anterior region to $1.4 \mathrm{~mm}$ in the posterior. It is also thicker on the facial side than on the lingual side and has been cited as 1.12 to $1.33 \mathrm{~mm}$ in the maxilla and 1.25 to $2.98 \mathrm{~mm}$ for mandible with thickness tending to increase from the cementoenamel junction to the apex. ${ }^{19}$ We used 'linear probe' on the extraoral surface of periapical area of the concerned teeth. In cases of thick buccal cortical bone, an approach from the lingual/palatal side on the periapical area of the concerned teeth through a 'transcavitary probe' might prove effective. This approach might obviate the issue of increased thickness encountered on buccal side. Notwithstanding that clinician's experience in performing USG and his/her knowledge of oral anatomy and pathology are important in the correct examination and interpretation of USG and color Doppler images.

Efficacy of USG in distinguishing different types of cysts like developmental or traumatic were evaluated by Cotti and Campisi. ${ }^{13}$ They cited limitations in differentiating varied types of cysts specifically. Sumer et $\mathrm{a}^{11}$ also concurred to this view which was in agreement to our findings.

\section{CONCLUSION}

The study demonstrated that USG in combination with color Doppler is a reliable method for the differential diagnosis of solid and cystic lesions of pulpal origin. Good correlations existed between the echo intensity and histopathologic features of periapical cyst and granuloma. Color Doppler was found effective in diagnosing solid lesions, like periapical granuloma, while along with USG it could very well differentiate noninfected and infected cysts. Overall, cysts had $100 \%$ correlation with its histopathology but granuloma had only $70 \%$. The thick overlying bone was the main reason hindering ultrasonography of intrabony periapical lesion. With sound knowledge of oral anatomy/histology and basic expertise in USG and color Doppler, the method offers a safe and noninvasive diagnostic option in dentistry.

\section{REFERENCES}

1. Nair PN. Pathogenesis of apical periodontitis and the causes of endodontic failures. Crit Rev Oral Biol Med 2004;15:348-381.

2. Lauria L, Curi MM, Chammas MC, Pinto DS, Torloni H. Ultrasonography evaluation of bone lesions of the jaw. Oral Surg Oral Med Oral Pathol Oral Radiol Endod 1996;82:351-357.
3. Nobuhara WK, Del Rio CE. Incidence of periradicular pathoses in endodontic treatment failures. J Endod 1993;19:315-318.

4. Moore CL, Copel JA. Point-of-care ultrasonography. N Engl J Med 2011;364:749-757.

5. Arger PH, Schultz SM, Sehgal CM, Cary TW, Aronchick J. Teaching medical students diagnostic sonography. J Ultrasound Med 2005;24:1365-1369.

6. Zhao Y, Ariji Y, Gotoh M, et al. Colour Doppler sonography of the facial artery in the anterior face. Oral Surg Oral Med Oral Pathol Oral Radiol Endod 2002;93:195-201.

7. Barber FE, Lees S, Lobene RR. Ultrasonic pulse-echo measurements in teeth. Arch Oral Biol 1969;14:745-760.

8. Tsiolis FI, Needleman IG, Griffiths GS. Periodontal ultrasonography. J Clin Periodontol 2003;30:849-854.

9. Emshoff R, Bertram S, Rudisch A, Gassner R. The diagnostic value of ultrasonography to determine temporomandibular joint disc position. Oral Surg Oral Med Oral Pathol Oral Radiol Endod 1997;84:688-696.

10. Kleinheinz J, Anastassov GE, Joos U. Ultrasonographic versus conventional diagnostic procedures in dislocated subcondylar mandibular fractures. J Craniomaxillofac Trauma 1997;3: 40-42.

11. Sumer AP, Danaci M, Ozen Sandikci E, Sumer M, Celenk P. Ultrasonography and Doppler ultrasonography in the evaluation of intraosseous lesions of the jaws. Dentomaxillofac Radiol 2009;38:23-27.

12. Cotti E, Campisi G, Ambu R, Dettori C. Ultrasound real-time imaging in the differential diagnosis of periapical lesions. Int Endod J 2003;36:556-563.

13. Cotti E, Campisi G. Advanced radiographic techniques for the detection of lesions in bone. Endodontic Topics 2004;7:52-72.

14. Gundappa M, Ng SY, Whaites EJ. Comparison of ultrasound, digital and conventional radiography in differentiating periapical lesions. Dentomaxillofac Radiol 2006;35:326-333.

15. Goel S, Nagendrareddy SG, Raju MS, et al. Ultrasonography with colour Doppler and power Doppler in the diagnosis of periapical lesions. Indian J Radiol Imaging 2011;21:279-283.

16. Schwartz-Dabney CL, Dechow PC. Variations in cortical material properties throughout the human dentate mandible. Am J Phys Anthropol 2003;120:252-277.

17. United Nations Scientific Committee on the effects of Atomic Radiation (UNSCEAR) 2008. Report to the general assembly, with scientific annexes. Sources and effects of ionizing radiation. Volume 1: Annex A: Medical Radiation Exposures.

18. Gibbs SJ. Effective dose equivalent and effective dose: Comparison for common projections in oral and maxillofacial radiology. Oral Surg Oral Med Oral Pathol Oral Radiol Endod 2000;90:538-545.

19. Park J, Cho HJ. Three-dimensional evaluation of interradicular spaces and cortical bone thickness for the placement and initial stability of microimplants in adults. Am J Orthod Dentofac Orthop 2009;136:314.e1-314.e12. 